\title{
Inhibition of Nuclear Envelope Reconstitution in Xenopus Interphase Egg Extract by Hemin
}

\author{
Ryutarou Nishiyama $^{1}$, Mayumi Ikami ${ }^{1}$, Fumiaki Yamao ${ }^{2}$, Sumiko Tsuchida ${ }^{1}$, and Tatsuo Yagura*1 \\ ${ }^{1}$ Laboratory of Life Science, Department of Chemistry, Faculty of Science, Kwansei Gakuin University, \\ Nishinomiya, Hyogo 662-8501, Japan, and ${ }^{2}$ National Institute of Genetics, Mishima, Shizuoka 411-8540, Japan
}

pseudonuclei/Xenopus/egg extract/hemin/nuclear envelope

\begin{abstract}
Addition of hemin to the nuclear reconstitution system of Xenopus interphase egg extract using sperm head chromatin resulted in abnormal pseudonuclei exhibiting flattened membrane patches randomly distributed both on the surface and inside the nuclei. The structures that resembled nuclear pores were observed on these flattened membrane patch structures. Although the nucleosome structure was formed as revealed by the micrococcal nuclease digestion, the B-type lamin uptake into the nuclei was inhibited by hemin. Using heminagarose affinity chromatography, we isolated several hemin-binding proteins from fully reconstituted pseudonuclei. Some of the hemin-binding proteins bound concanavalin A (Con A). Comparison of hemin-binding proteins with those isolated from both fractions of supernatant and pellet separated by high speed centrifugation of the egg extract showed that the hemin-binding proteins of pseudonuclei were supplied from both fractions. The uptake of nuclear hemin-binding proteins did not occur in the incompletely reconstituted nuclei resulting from addition of excess sperm chromatin to the system. These results suggest that the hemin-binding proteins participate in the late steps of nuclear reconstitution during formation of the nuclear envelope.
\end{abstract}

The structure of nuclei in higher eukaryotes changes dramatically during the cell cycle, especially during the mitotic phase. Following these changes of nuclear structure, the organization of the genome is correctly restored. Although much information has emerged as to individual phenomena during nuclear reconstitution after mitosis of cells, how component proteins of the nucleus interact to form a functioning nucleus is still unknown. Difficulties emerge when using whole cells as test material. To overcome these difficulties, a cell-free nuclear reconstitution system derived from Xenopus interphase eggs has been employed $(3,18)$. Studies have shown that the nuclear reconstitution system of Xenopus egg extract provides a suitable system for investigating various aspects of nuclear reconstitution (2).

By applying biochemical approaches to the detailed process involved in the assembly of protein components into the nuclei, the assembly processes of histones, lamin, nuclear pore proteins, and DNA replication proteins have been studied $(1,5,11,15,17,26)$. Nevertheless, it has been speculated that many other proteins which might play key roles in the reconstitution process of nuclei await to be found by new methods. In this study, we have used hemin to elucidate the role of a new class of protein involved in the reconstitution process of the nuclear envelope of psuedonuclei from sperm chromatins in Xenopus interphase egg extract. Hemin is a versatile regulatory molecule known to regulate a variety of cellular processes such as differentiation, transcription, post-transcriptional processing, and protein degradation in a variety of systems (4, $6,8,20,24,34)$. Hemin interacts with biological membrane $(10,27)$ and the proteins in the cell membranes of bacteria and red cells $(21,29)$ possibly by its hydrophobic nature originating from porphyrines. Thus, using it, we expect to find a new class of proteins embedded in the nuclear membrane which participates in nuclear envelope reconstitution at anaphase of mitosis. Here we document that hemin induces incomplete nuclear envelope reconstitution of sperm head chromatin in Xenopus interphase egg extract possibly by binding to a special class of proteins. Upon observation of the detailed structure of chromatin reconstituted in the presence of hemin, we were able to characterize the step of the reconstitution process in which the hemin-binding proteins are incorporated into the reconstituting chromatin.

\footnotetext{
* To whom correspondence should be addressed.

Tel: +81-798-54-6406, Fax: +81-798-51-0914

E-mail: z88012@kgupyr.kwansei/ac.jp
} 


\section{MATERIALA AND METHODS}

\section{Reagents}

Hemin and hemin-agarose were purchased from Wako Pure Chemical Industries, Ltd. and Sigma, respectively. Hemin was dissolved in solution A $(16 \mathrm{mM} \mathrm{KCl} / 20 \mathrm{mM}$ Tris- $\mathrm{Hcl}, \mathrm{pH}$ 7.8) and stocked at $-20^{\circ} \mathrm{C}$ until use.

\section{Preparation of egg extract and sperm chromatin}

Demembranated sperm head chromatins were prepared from Xenopus testis and stored frozen at $-80^{\circ} \mathrm{C}$ until use as described previously (3). Extracts from Xenopus interphase eggs were prepared as described previously (30), rapidly frozen, and stored at $-80^{\circ} \mathrm{C}$ until use.

\section{Nuclear assembly assay}

Assays were performed as described previously (13). Aliquots of interphase extract were mixed with reagents plus demembranated sperm chromatins. A typical reaction consisted of $20 \mu \mathrm{l}$ extract, $2 \mathrm{mM}$ ATP, $20 \mathrm{mM}$ phosphocreatine, $50 \mu \mathrm{g}$ $/ \mathrm{ml}$ creatine phosphokinase, and sperm nuclei $(1,000 / \mu 1$ reaction mixture). Reaction mixtures were incubated at $22^{\circ} \mathrm{C}$ for the indicated times.

\section{Electron microscopy}

After incubation for the indicated times a $60 \mu \mathrm{l}$ aliquot of egg extract containing sperm chromatins was added to $1 \mathrm{ml}$ of 5 $\mathrm{mM}$ sodium phosphate ( $\mathrm{pH} 7.4)$ containing $2 \%$ glutaraldehyde. After further incubation for $3 \mathrm{~h}$ at $4{ }^{\circ} \mathrm{C}$, a pellet was obtained after centrifugation at $1,500 \times \mathrm{g}$ for $5 \mathrm{~min}$. The pellet was processed for electron microscopy as previously described (32).

Depletion of hemin-binding protein from the egg extract by hemin-agarose

Hemin agarose (Sigma) pre-equilibrated with MW buffer (500 $\mathrm{mM}$ sucrose, $2 \mathrm{mM} \mathrm{MgCl}$, $50 \mathrm{mM} \mathrm{KCl,} 10 \mathrm{mM}$ Hepes- $\mathrm{KOH}$, $\mathrm{pH}$ 7.6) was added to the egg extract $(1: 10, \mathrm{v} / \mathrm{v})$ and the mixture was incubated for $10 \mathrm{~min}$ at $4^{\circ} \mathrm{C}$ with gentle shaking. After removal of beads by centrifugation at $1,000 \mathrm{rpm}$ for 3 min at $4^{\circ} \mathrm{C}$, the sperm chromatin $(250 / \mu 1$ extract $)$ were added and incubated for $2 \mathrm{~h}$ at $22^{\circ} \mathrm{C}$ to test the reconstitution of pseudonuclei.

\section{Isolation of hemin-binding proteins with use of hemin- agarose}

Hemin-binding proteins from the Xenopus interphase egg extracts and the reconstituted nuclei were isolated using a hemin-agarose affinity resin according to the methods described earlier (21). Hemin-binding proteins were resolved by SDSPAGE and stained by the silver staining method.

Immunoblot analysis of lamin and concanavalin A-binding protein

SDS-gel electrophoresis, immunoblotting to PVDF mem- brane, and detection of proteins were performed essentially as previously described $(22,23)$. The lamin which was transferred to PVDF membrane was allowed to react with anti-lamin $\mathrm{B}_{1}$ monoclonal antibody (Lamin B (Ab-1), Calbiochem) followed by alkaline phosphatase conjugated rabbit anti-mouse IgG antibody. For analysis of concanavalin A-binding protein, the proteins isolated from the reconstituted pseudonuclei were separated by SDS-gel electrophoresis, blotted onto PVDF membrane and detected using biotin-Con A (Seikagaku Co.) and streptoavidine-alkaline phosphatase (Life Technologies).

\section{Assay for DNA systhesis}

Sperm head chromatins $(1,000 / \mu 1$ extract $)$ were added to interphase egg extract containing ATP and ATP-regenerating system along with $\left[{ }^{3} \mathrm{H}\right] \mathrm{TTP}(0.3 \mu \mathrm{Ci} / \mu \mathrm{l}$ extract; $81.3 \mu \mathrm{Ci}$ $/ \mathrm{mmol}$, NEN Research Products) and incubated for the indicated time at $22^{\circ} \mathrm{C}$. After incubation each reaction mixture was spotted onto a glass filter and processed as described earlier (30).

Analysis of nucleosome structure by the use of micrococcal endnuclease digestion method

Sperm chromatins were incubated in $1 \mathrm{ml}$ of the egg extract containing ATP and ATP regenerating system for $2 \mathrm{~h}$ at $22^{\circ} \mathrm{C}$. Then, the mixture was diluted with three volumes of buffer I (0.34 M sucrose, $15 \mathrm{mM}$ Tris- $\mathrm{HCl}, \mathrm{pH} 7.4,15 \mathrm{mM}$ $\mathrm{KCl}, \quad 0.5 \mathrm{mM}$ spermidine, $0.15 \mathrm{mM}$ spermine, $0.2 \mathrm{mM}$ EDTA, protease inhibitor cocktail (Sigma)) and centrifuged at $8,000 \times \mathrm{g}, 4^{\circ} \mathrm{C}$ for $10 \mathrm{~min}$. The pelleted chromatin was washed successively with buffer I and buffer II (10 mM Tris$\mathrm{HCl}, \mathrm{pH} 8,1 \mathrm{mM}$ EDTA, $100 \mathrm{mM} \mathrm{NaCl}$ ). The washed chromatin was suspended in $10 \mu l$ of buffer II and then processed for the analysis by the micrococcal endnuclease digestion method described previously (16).

\section{RESULTS}

Inhibition of swelling of sperm chromatin by hemin The addition of hemin to Xenopus interphase egg extract resulted in the inhibition of reconstitution of sperm head chromatin in a concentration dependent manner. We classified the shape of chromatin observed in the preparations, for convenience, into three types: Type 1 is fully swollen chromatin corresponding to normally formed pseudonuclei; Type 2 is rod-shaped decondensed chromatin; and Type 3 is round shape but not swollen (Fig. 1). As described earlier (30), sperm chromatin is unpacked in two main steps referred to as decondensation and swelling. The decondensation step is a nucleoplasmin-mediated reaction producing rodshaped chromatin, and the second swelling step is nuclear envelope-dependent swelling resulting in round pseudonuclei capable of DNA replication. Type 1 is typical of chromatin after the second step of nuclear 
formation, and Type 2 closely resembles chromatin at the decondensation step. On the other hand, Type 3 was scarcely observed on the non-inhibited reconstitution process of pseudonuclei. The ratio of Type 1 decreased by increasing the hemin concentration and disappeared at concentration greater than $100 \mu \mathrm{M}$ hemin (Fig. 2). Inversely, the ratio of Type 2 increased first and then declined rapidly but the ratio of Type 3 increased almost linearly as hemin concentration increased. At 200 $\mu \mathrm{M}$ hemin concentration, approximately $80 \%$ of chromatin became Type 3 after $2 \mathrm{~h}$ incubation.

To examine the process of the changes in form of chromatin in the presence of hemin in more detail, the time course of the changes was examined. As shown in Fig. 3, Type 2 appeared within the initial $5 \mathrm{~min}$ of the incubation period but declined rapidly with time. On the other hand, the ratio of round-shaped Type 3 chromatin increased as if taking the place of Type 2 chromatin. Fig. 4 shows representative chromatins observed after the indicated incubation time periods, compared with controls. In the control incubation, de-
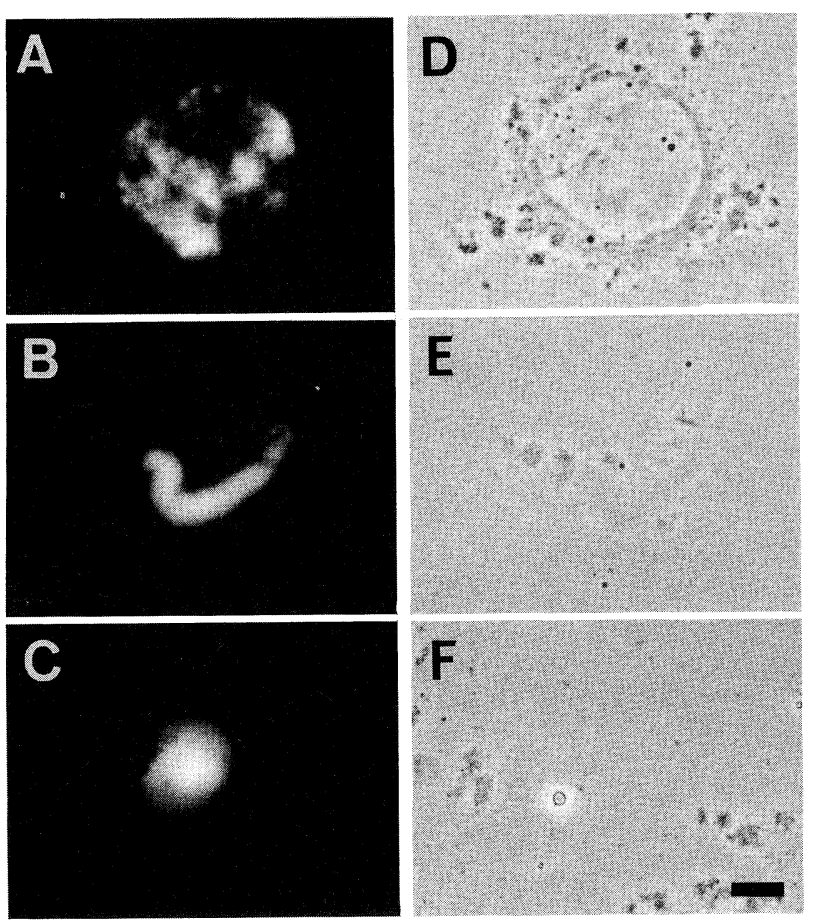

Fig. 1. Chromatin conformations observed in preparations of the interphase egg extract incubated with hemin. Nuclear assembly assays were performed as described previously (13) in the presence or absence of hemin (Wako Co. Ltd.). The typical conformations of chromatin were classified into three types (see text). Type 1, A and D; Type 2, B and E; Type 3, C and F. The same fields are shown using epifluorescence optics of Hoechst $33258(\mathrm{~A}-\mathrm{C})$ or phase contrast (DF). The boundary of surface region of the round chromatin shown in $\mathrm{F}$ is not clear, indicating a deficiency of continuous nuclear membrane. Bar, $10 \mu \mathrm{m}$.

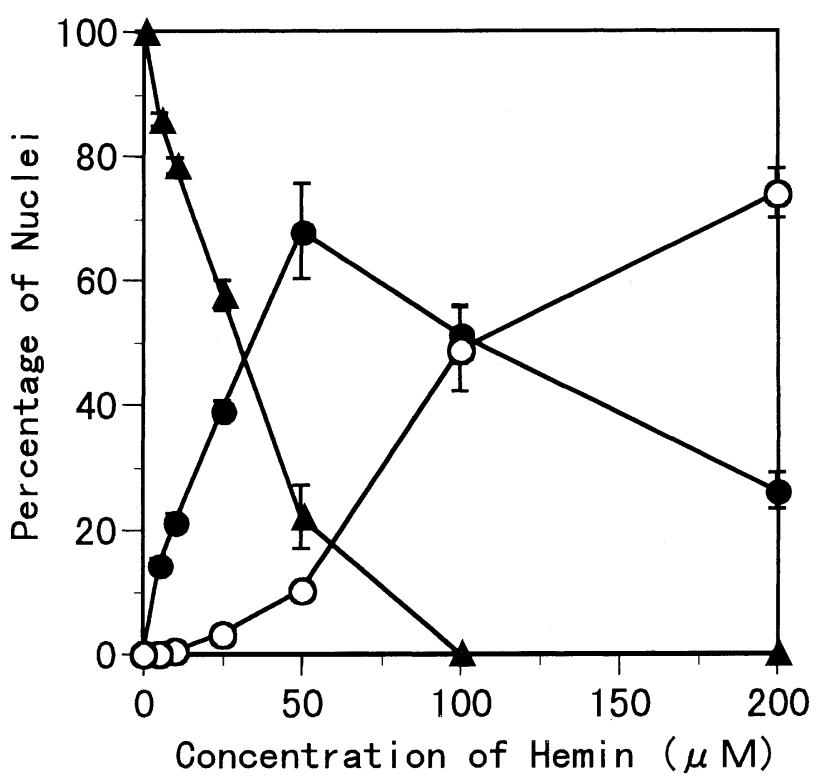

Fig. 2. Effect of hemin on nuclear reconstitution. Hemin was added at the start of incubation of sperm chromatin in egg extract at the concentrations indicated $(1 \mu \mathrm{l}$ hemin solution/13.5 $\mu$ l reaction mixture). After $2 \mathrm{~h}$ incubation at $22^{\circ} \mathrm{C}$, the sperm chromatins were fixed/stained in buffer containing 3.7\% formaldehyde and $20 \mu \mathrm{g} / \mathrm{ml}$ Hoechst fluorescent dye 33258 (13), and then the ratio of each type was determined. Classification of each type was according to that shown in Fig. 1. $\boldsymbol{\Delta}$, Type 1; 0 , Type 2; $\bigcirc$, Type 3.

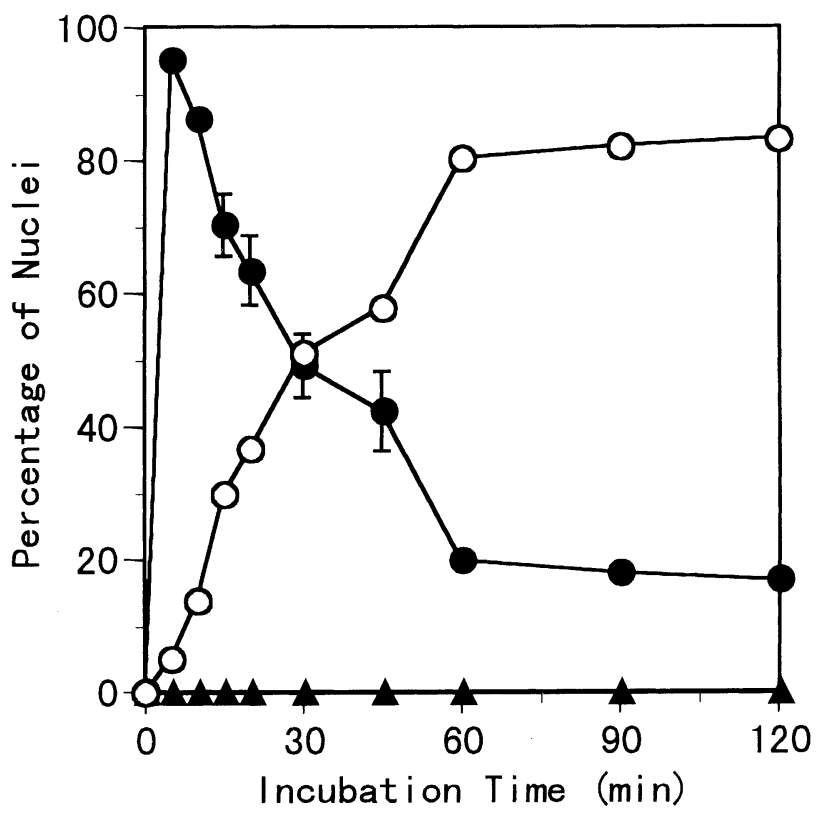

Fig. 3. Changes in the ratio of each type of chromatin during hemin inhibition. Type and its ratio of sperm chromatin were determined at the times indicated during hemin inhibition at $200 \mu \mathrm{M}$ in the egg extract as described in the legend to Fig. 2. $\boldsymbol{\Delta}$, Type 1; $\mathbf{O}$, Type $2 ; \bigcirc$, Type 3 . 

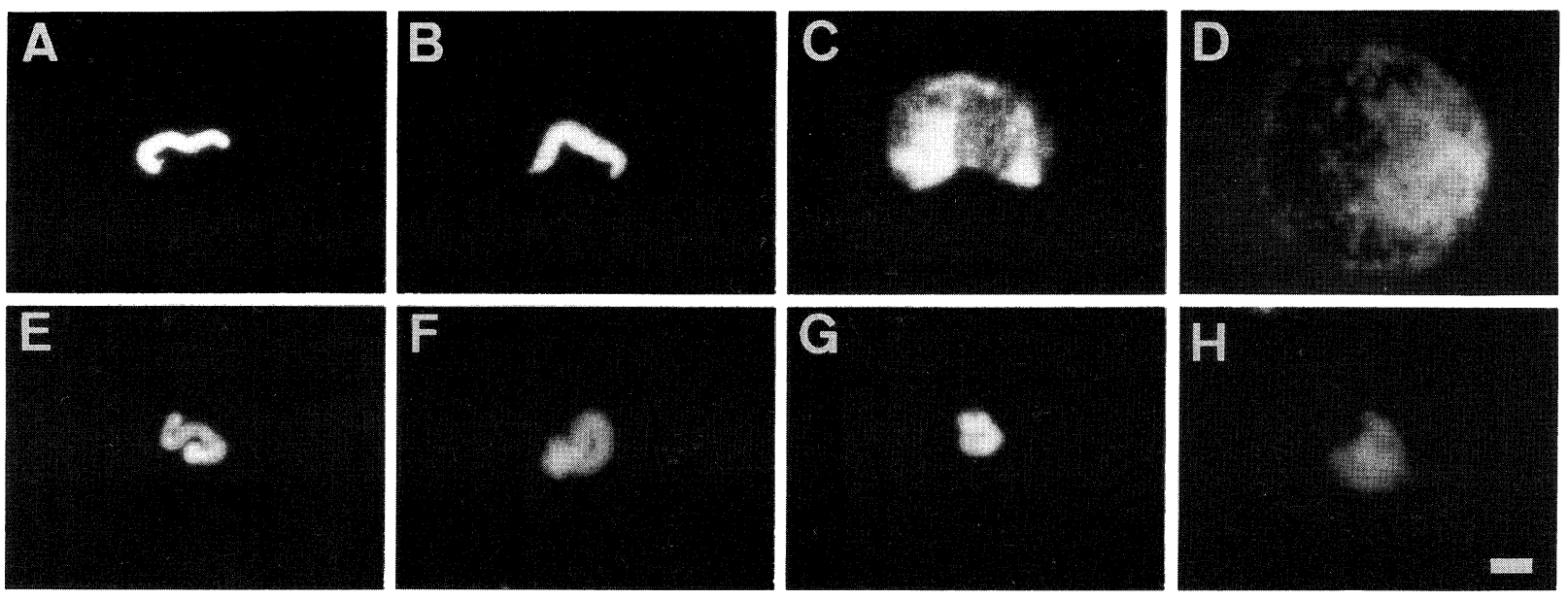

Fig. 4. Changes in the conformation of sperm chromatin during hemin inhibition observed with fluorescent microscopy. Sperm chromatin was incubated in egg extract at $22^{\circ} \mathrm{C}$ in the absence (A-D) or presence (E-H) of $200 \mu \mathrm{M}$ hemin. At the times indicated below, the chromatin was fixed/stained as described in the legend to Fig. 2, and then representative forms for each incubation time were photographed. Incubation times were; (A, E), $10 \mathrm{~min}$; (B, F), $20 \mathrm{~min}$; (C, G), $45 \mathrm{~min}$; (D, H), $120 \mathrm{~min}$. Bar, $10 \mu \mathrm{m}$.

condensed rod-like chromatins swelled at multiple sites outward to form a round pseudonuclei with sparsely distributed DNA. In contrast to the control chromatin, the hemin-treated chromatin did not swell but displayed lithe movement resulting in bends in roundcompact chromatin (Fig. 4E to $\mathrm{H}$ ). Upon detailed observation of chromatins during hemin treatment, we found that the rod-like decondensed chromatins (Type 2) became folded to form Type 3 chromatin in the presence of hemin.

\section{No DNA synthesis was detected in hemin-blocked sperm chromatin}

DNA in pseudonuclei formed in the egg extract begins to replicate during the early phase of swelling (30). This reaction is one of the indexes for perfection of the reconstitution in nuclear matrix region. Fig. 5 shows the effect of hemin on DNA synthesis. Hemin above $50 \mu \mathrm{M}$ concentration inhibited DNA synthesis completely. Partial DNA synthesis in the chromatin was not observed at these concentrations of hemin in another experiment using BrdUTP and cytochemical method (30) (data not shown). Thus the reconstitution of nuclear structure responsible for DNA replication failed to occur in the presence of hemin at above $50 \mu \mathrm{M}$.

The flattened membrane patches in sperm chromatin incubated in a hemin-blocked preparation

Contrast optics microscopy of the Type 3 sperm chromatin (Fig. 1F) showed an absence of the phase dark structures which are diagnostic of nuclear envelope assembly suggesting the lack of nuclear membranes. To examine whether the nuclear envelope had indeed not formed in the presence of hemin, the ultrastructure of the chromatins was examined at several incubation period. We used hemin at a concentration of $50 \mu \mathrm{M}$ since the sperm chromatins incubated in the presence of hemin at more than $100 \mu \mathrm{M}$ were too fragile to sample for electron microscopy. Fig. 6A shows one decondensed chromatin, and one decondensing chromatin with unpacked region as indicated by the arrow after $10 \mathrm{~min}$ incubation in the presence of hemin. Within 20 min incu-

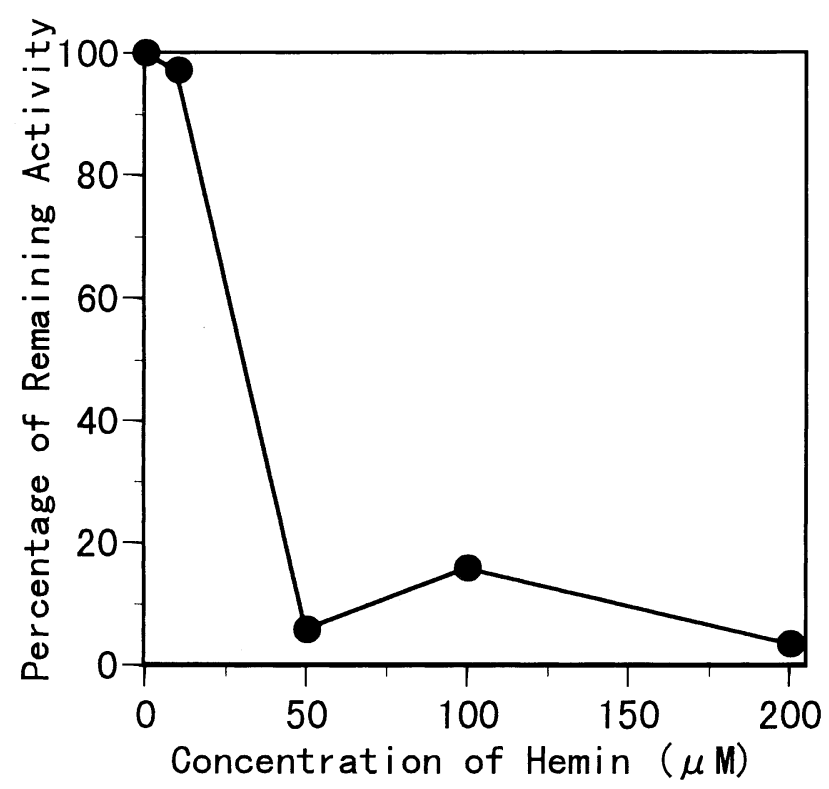

Fig. 5. Inhibition of DNA synthesis in the chromatin by hemin treatment. Sperm chromatin was incubated in the egg extract at $22^{\circ} \mathrm{C}$ containing $\left[{ }^{3} \mathrm{H}\right] \mathrm{TTP}$ plus hemin at the indicated concentrations. After $2 \mathrm{~h}$ incubation, $10 \mu \mathrm{l}$ aliquots were withdrawn to determine incorporated [3]TMP. Value for $100 \%$ activity was $7,600 \mathrm{cpm}$. 

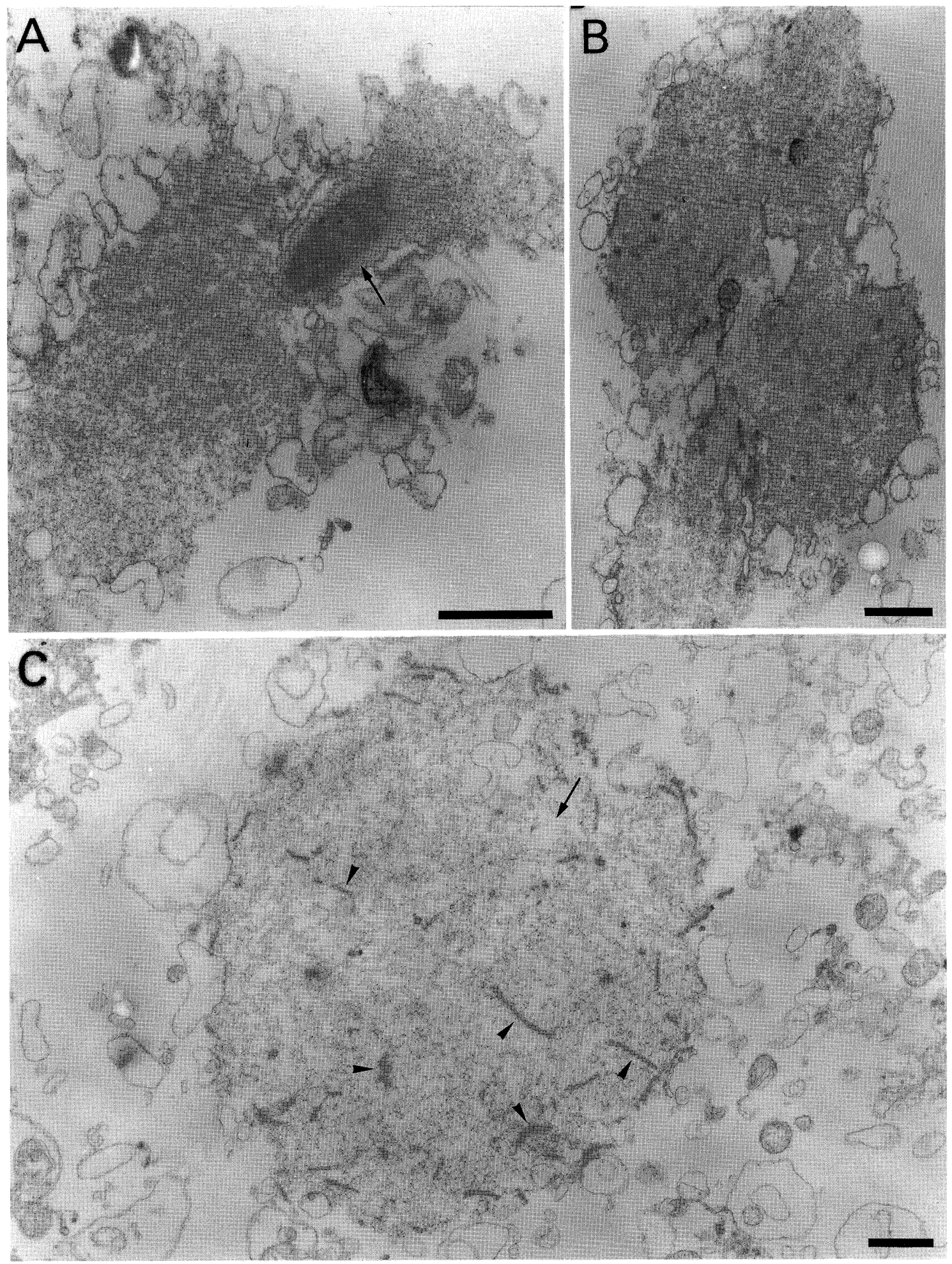

Fig. 6. Ultrastructure of hemin-blocked chromatin. Sperm chromatin was incubated in the egg extract in the presence of $50 \mu \mathrm{M}$ hemin. At 10 $\min (\mathrm{A}), 20 \mathrm{~min}$ (B) or $120 \mathrm{~min}$ (C) incubation period, the chromatin was processed for electron microscopy as described under Materials and Methods. In A, two chromatins are evident, one is a decondensing sperm head (indicated by arrow). In C, numerous double-layered nuclear membranes are shown inside the chromatin (several of them indicated by arrow heads). Arrow in C shows loose cleft. Bars, $1 \mu \mathrm{m}$. 

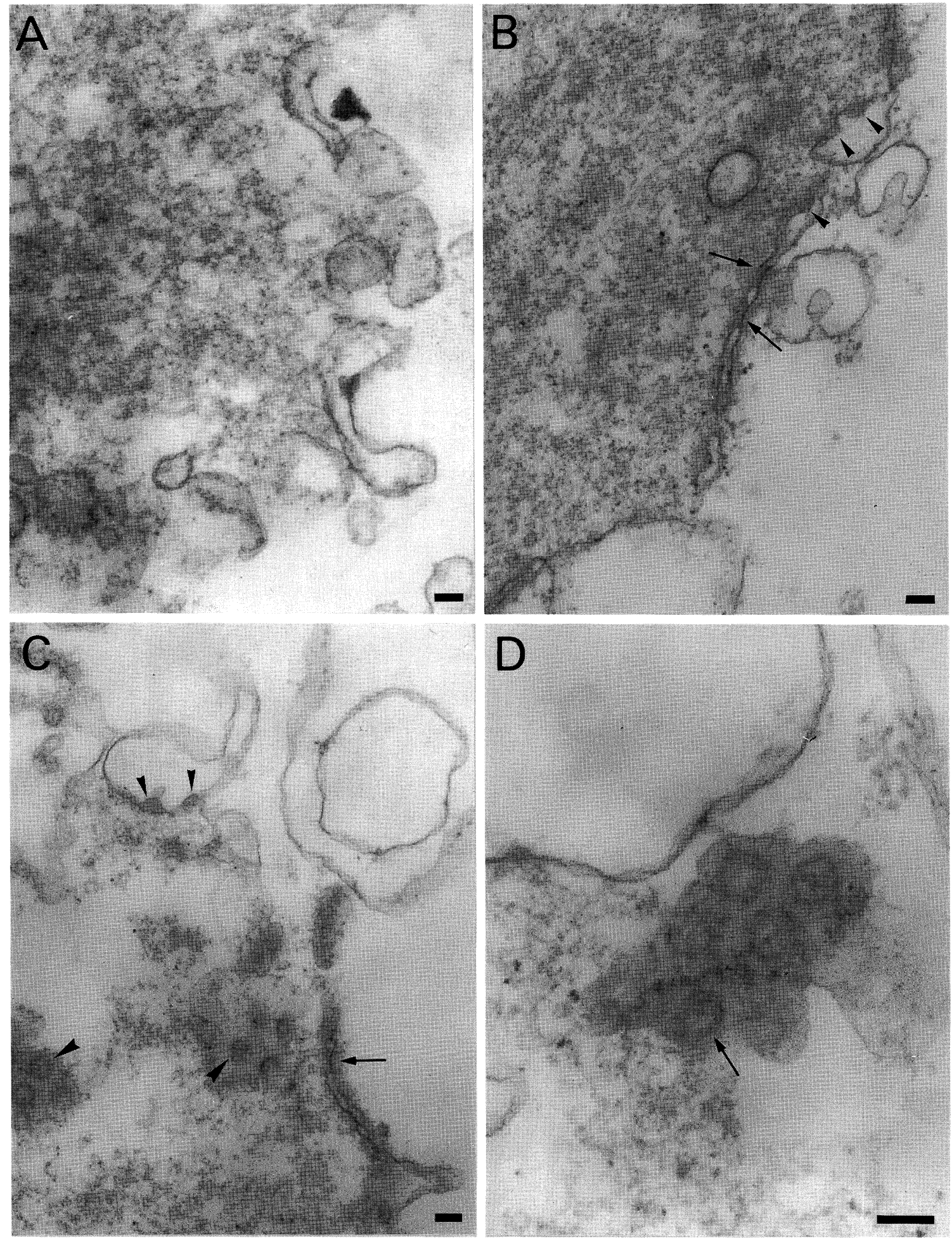

Fig. 7. Process of formation of double-layered nuclear membrane and nuclear pore in hemin-blocked chromatin. Sperm chromatin was incubated in the egg extract in the presence of $50 \mu \mathrm{M}$ hemin. After $10 \mathrm{~min}(\mathrm{~A}), 20 \mathrm{~min}(\mathrm{~B})$ or $120 \mathrm{~min}(\mathrm{C}, \mathrm{D})$ incubation period, the chromatin was processed for electron microscopy as described under Materials and Methods. In B and C, nuclear pores of the double-layered nuclear membranes and pre-nuclear pore-like structure are shown (arrows, nuclear pores; small arrowheads, pre-nuclear pore-like structure). In C and D, cross section images of nuclear pores are shown (large arrowheads). The cross section image of a nuclear pore observed in D (indicated by arrow) shows the representative structure of nuclear pore complex. Bars, $0.1 \mu \mathrm{m}$. 
bation period, all the sperm chromatins transformed to the decondensed form as shown in Fig. 6B, although numerous membtane vesicles attached but did not fuse to each other to form a double-layered nuclear membrane on the majority of sperm chromatins. After $2 \mathrm{~h}$ incubation, these membrane vesicles fused onto the hemin-blocked chromatins to form the flattened membrane structures (Fig. 6C), but, strikingly, these membrane structures were not continuous. The membrane structures appeared as patches on the surface of chromatins with some of the patches actually enfolded in the inside region of the sperm chromatins. Most of sperm chromatins were of this sort, which was scarcely observed in the non-blocked control preparation. Folded chromatin such as that shown in Fig. 6B and the round chromatin with a loose cleft (arrow in Fig. 6C) were often observed in the hemin-blocked preparation. On the basis of these observations and those in Fig. 4 of curved sperm chromatin, we speculated that some of the membrane patches became engulfed inside the nucleus during the folding movement of the chromatin.

Next, to pursue how these membrane patches were formed and whether they carried nuclear pores, we examined the time course of formation of membrane patches along with its nuclear pore at the ultrastructural level. At 10 min incubation, numerous membrane vesicles attached to the surface of chromatins, but no nuclear pore was observed (Fig. 7A). As shown in Fig. $7 \mathrm{~B}$, part of the membrane vesicles flattened to form membrane patches with numerous nuclear pore-like structures (arrows in Fig. 7B) after 20 min incubation. After $2 \mathrm{~h}$ incubation, all of the flattened membrane patches possessed this pore-like structure (Fig. 7C). Furthermore, structures that protruded inside the membrane vesicles on the side attached to chromatin were observed (Fig. 7B and C, arrowheads). This structure closely resembled the pre-nuclear pores reported by Sheehan et al. (28). A section tangential to the nuclear pore-like structures of the membrane patches (Fig. 7D) show they consisted of representative structural components as reported elsewhere for nuclear pore complex $(14,25)$. Eight multi-domain spokes to yield a doughnut hole in the double membrane can be counted on the pore indicated by arrow. Thus, the flattened membrane patches are suggested to be associated with the nuclear pore complex but it is uncertain whether the pores were completely constructed in the hemin-inhibited preparation. Fig. 8 shows the enlarged figure of an enfolded membrane structure. Several pores are also visible on the membrane but no difference was found from those that existed on the surface of the chromatin.

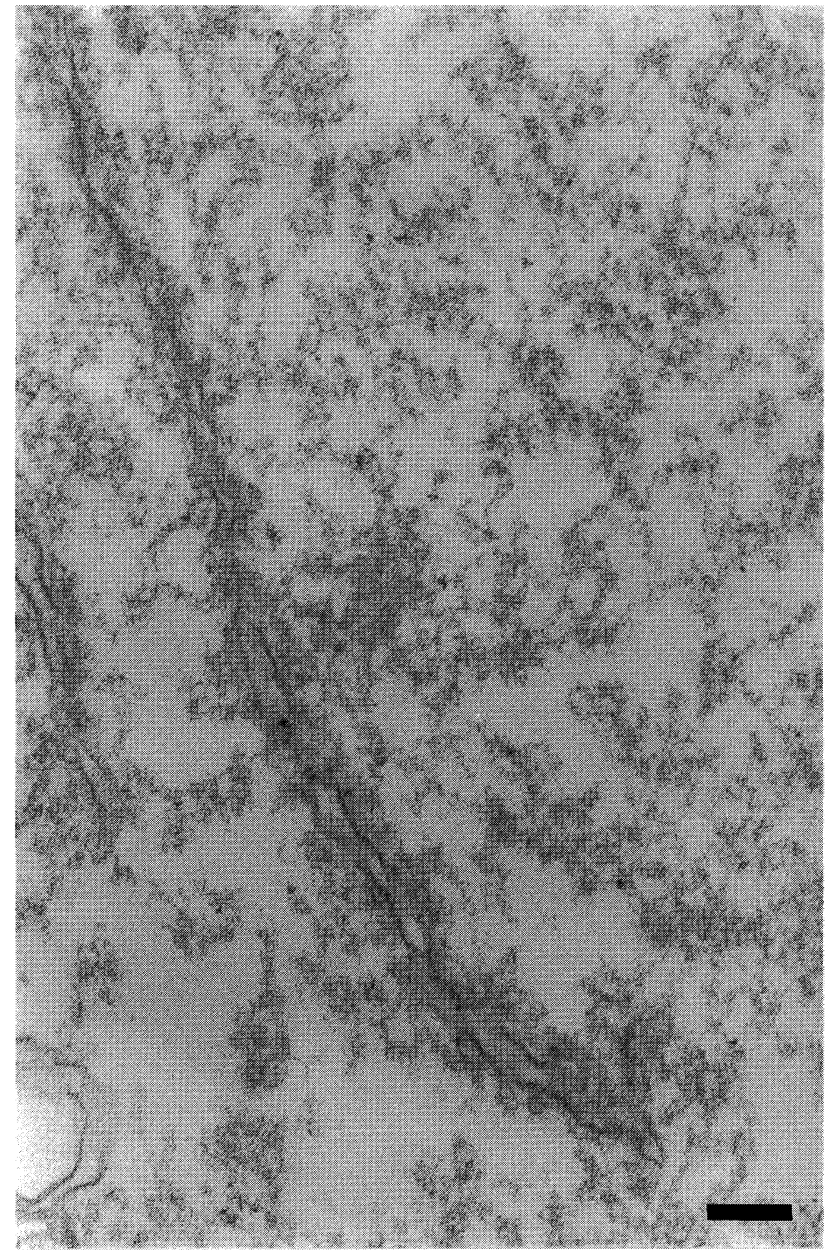

Fig. 8. Double-layered nuclear membrane enfolded in heminblocked chromatin. Preparation for electron microscopy was made as described in the legend to Fig. 6. The ultrastructure of one of the nuclear membranes inside the nuclei is shown. Numerous nuclear pores are observed within the nuclear membrane, but the face and back of the membrane are not distinguishable. Bar, $0.1 \mu \mathrm{m}$.

\section{Inhibition of the incorporation of the B-type lamin into sperm chromatins by hemin}

As shown in Fig. 9, the amount of B-type lamin incorporated into the hemin-blocked chromatin was nearly the same or below the amount of B-type lamin incorporated into wheat germ agglutinin (WGA)-blocked chromatins. WGA prevents a portion of nuclear pore assembly from forming (7), resulting in the inhibition of uptake of the B-type lamin LIII through nuclear pores $(5,31)$. Thus, the results of Fig. 9 indicated that the Btype lamin incorporation into the chromatins was severely inhibited by hemin. In the presence of hemin, the chromatin could reconstitute the double-layered nuclear envelope-like membrane patches but it did not incorporate the B-type lamin, suggesting some defects of 


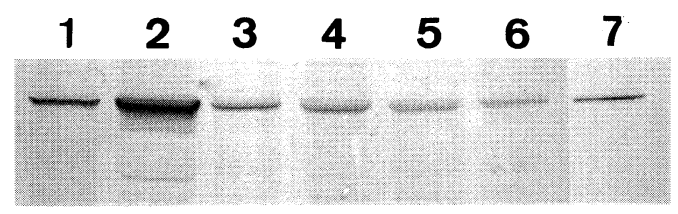

Fig. 9. Western immunoblotting of lamin incorporated into hemin-blocked chromatin. After incubation in the egg extract containing hemin at the concentrations indicated for $2 \mathrm{~h}$, sperm chromatin was isolated and purified by centrifugation. The lysate of chromatins was analyzed by western immunoblotting procedure using the anti-lamin $\mathbf{B}_{1}$ antibody (Lamin B (Ab-1), Calbiochem). Lane 1, egg extract; lane 2, pseudonuclei (no hemin control); lane 3, $50 \mu \mathrm{M}$ hemin; lane 4, 100 $\mu \mathrm{M}$ hemin; lane 5, $200 \mu \mathrm{M}$ hemin; lane 6, $300 \mu \mathrm{M}$ hemin; lane 7, 1 $\mathrm{mg} / \mathrm{ml}$ WGA. Lamin B (Ab-1) recognized a protein band of approximately $66 \mathrm{kDa}$ in the immunoblots of all the preparations.

construction of either the nuclear envelope or nuclear matrix structure.

\section{Nucleosome structure was formed under inhibition by hemin}

DNA fragments characteristically the size of a somatic mononucleosome monomer DNA and a ladder of periodic sized DNA fragments were protected from digestion by micrococcal nuclease in chromatin preparation

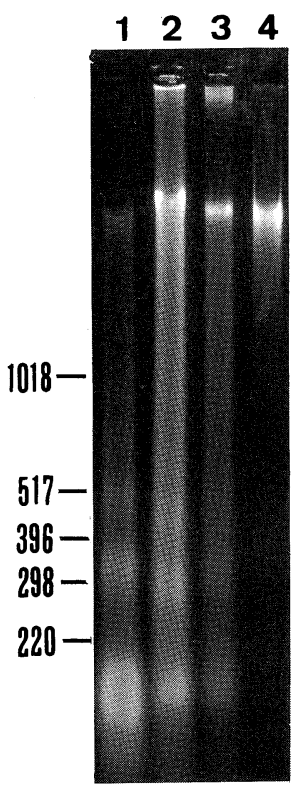

Fig. 10. Formation of nucleosome structure in hemin-blocked chromatin. Sperm chromatin was incubated in egg extract containing $200 \mu \mathrm{M}$ hemin for $2 \mathrm{~h}$ and then processed for analysis of nucleosome structure following micrococcal nuclease digestion and agarose gel electrophoresis as described under Materials and Methods. Lane 1 , nuclei isolated from mouse liver; lane 2, control pseudonuclei formed in the absence of hemin; lane 3, chromatin inhibited by 200 $\mu \mathrm{M}$ hemin; lane 4 , chromatin as in lane 1 with no digestion by micrococcal nuclease. incubated in the presence of hemin (Fig. 10). As reported by Philpott and Leno (26), nucleosome core formation is mediated by a gain of histones occurring at the decondensation step (this step was designated as the first stage of reconstitution in that report). The corresponding results (see Fig. 2) in which the addition of hemin inhibited the swelling of sperm chromatin but not its decondensation, indicate that hemin inhibits nuclear reconstitution after the step of decondensation.

\section{Nuclear reconstitution did not progress in egg extract depleted of hemin-binding proteins with hemin- agarose}

Hemin may bind to a variety of proteins in the egg extract through hydrophobic interactions. Thus, hemin might inhibit nuclear reconstitution by binding to specific proteins. To assess directly the participation of hemin-binding proteins in reconstitution, we depleted hemin-binding proteins from the egg extract with hemin-agarose beads, and tested for the capacity to reconstitute nuclei. As shown in Fig. 11, the egg extract depleted of hemin-binding proteins lost its ability to reconstitute pseudonuclei.

\section{Hemin-binding proteins transferred into the pseudo- nuclei}

To identify candidate proteins responsible for hemin

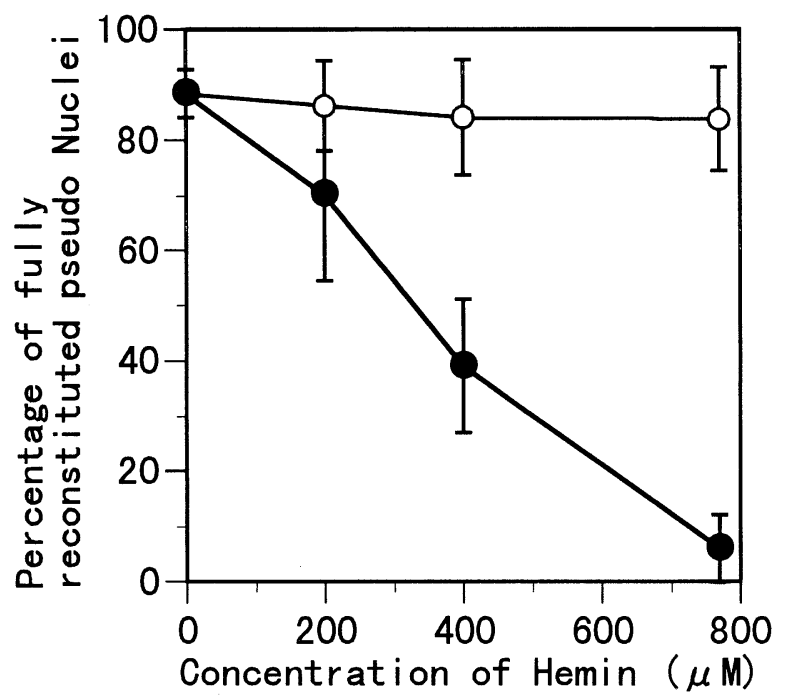

Fig. 11. Effect of depletion of hemin-binding proteins from the egg extract on reconstitution of pseudonuclei. Sperm chromatin was incubated in egg extract which had been depleted of hemin-binding proteins using hemin-agarose beads at various concentrations $(\boldsymbol{O})$ or mock-treated with agarose beads (Bio-Gel A) (BIO-RAD) at the same volumes as control $(\bigcirc)$ as described in Materials and Methods. After $2 \mathrm{~h}$ incubation, the formation of pseudonuclei was monitored employing Hoechst 33258 fluorescent staining procedure and determined the inhibition of nuclear reconstitution. 
inhibition, hemin-binding proteins were separated using hemin-agarose affinity chromatography. Several hemin-binding proteins were isolated by the batch method from both the supernatant and pellet fractions of egg extract (Fig. 12, lanes 1 and 2). Fig. 12, lane 3 shows hemin-binding proteins recovered from the pseudonuclei fully reconstituted after $2 \mathrm{~h}$ incubation. The major hemin-binding proteins of high molecular weight of the pseudonuclei were those with molecular masses of $150,140,105,60,58,57,54,51,47$ and 45 $\mathrm{kDa}$. On the basis of their molecular mass, we assumed that $140,105,54$ and $47 \mathrm{kDa}$ proteins were derived from the supernatant fraction and those of 60,57 , 51,47 and $45 \mathrm{kDa}$ were from the pellet fraction. The origin of the 150 and $58 \mathrm{kDa}$ proteins could not be identified. These proteins might have been actively transferred into the reconstituting nuclei because they were highly concentrated to the pseudonuclei although they were not major proteins in egg extract.

To determine when hemin-agarose binding proteins were transferred into the sperm chromatin, we examined the presence of hemin-binding proteins in sperm chromatin which were incompletely reconstituted. Incompletely reconstituted chromatin can be obtained by adding 10 -fold excess number of sperm chromatin to the reconstitution system without use of inhibitors. As the components necessary for nuclear reconstitution are depleted if excess chromatin are added, the chromatins cease their process of reconstitution. Under the condition used, most of the chromatin activity was terminated at the decondensation step. Fig. 12, lane 4 shows that only a small amount of hemin-binding proteins were transferred to the incompletely reconstituted chromatins although this preparation contained sperm chromatins 10-fold over those of the control preparation (compare with lane 3 ). The protein bands of 60,51 and $45 \mathrm{kDa}$ are barely visible. Thus, the results shown in Fig. 12 indicate that most of hemin-binding proteins were transferred into the chromatins after the decondensation step from both the supernatant and pellet fractions of egg extract.

\section{A part of hemin-binding proteins of the pseudonuclei bound Con $A$}

Reconstitution of intact continuous nuclear envelope was impaired by hemin as shown in Fig. 6. Thus it can be speculated that the hemin-binding proteins participate in the formation of the nuclear envelope and are an integral nuclear membrane protein. Numerous proteins localized to membrane fraction and nuclear pore complex have glycosyl moieties $(9,11,12$ for review), hence we performed lectin-binding analysis. On western-blotting analysis, none of the hemin-binding proteins bound to the biotin conjugated WGA (data not shown) but, as shown in Fig. 13, the hemin binding
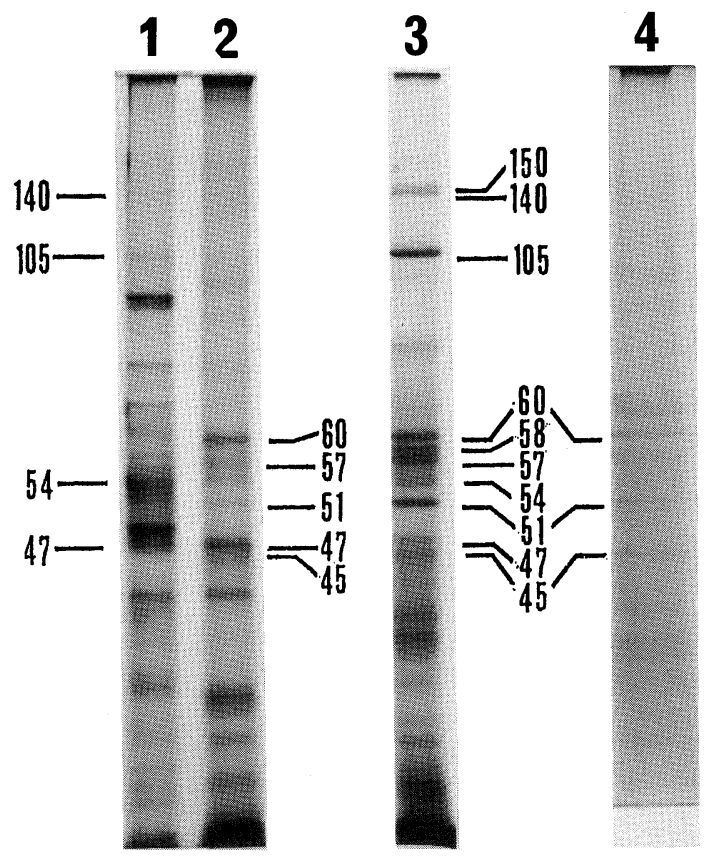

Fig. 12. Isolation of hemin-binding proteins of the pseudonuclei and determination of the step of their incorporation into pseudonuclei. Hemin-binding proteins were isolated from high-speed supernatant (lane 1) and pellet (lane 2) fractions of the egg extract, or from sperm chromatin which was incubated in egg extract for $2 \mathrm{~h}$ at $22^{\circ} \mathrm{C}$ (lane 3). The protein bands except for those described in the text varied either in staining or not staining or quantity on the SDS-gel. Thus, most of these protein bands may be of low affinity to hemin agarose or non-specific contamination. Lane 4 shows the hemin-binding proteins isolated from the chromatin terminated at the decondensation step by adding excess sperm chromatin to the egg extract $(10,000 / \mu 1$ egg extract). The gel strip in lane 4 was overstained to show a trace amount of proteins because almost no protein bands were visible after staining for the same time as in lane 3 .

proteins of 140, 105 and $60 \mathrm{kDa}$ bound to Con A. Further, additional $75 \mathrm{kDa}$ Con A-binding protein was observed although the amount recovered from the pseudonuclei was negligible (see Fig. 12, lane 3). These proteins thus have $\alpha$-D-mannopyranose and sterically related sugars as indicated by the Con A binding specificity. When Con A was added to the system, the swelling of sperm chromatin was completely inhibited at 0.5 $\mathrm{mM}$ concentration. However, distinct from the results obtained by hemin inhibition, a large portion of the sperm chromatin ceased at the step of rod-shaped decondensed chromatin (Type 2). The reason for this difference may be ascribed to the binding of Con A to some other non-hemin-binding proteins that are essential for nuclear reconstitution.

\section{DISCUSSION}

This study has demonstrated that hemin inhibits nu- 


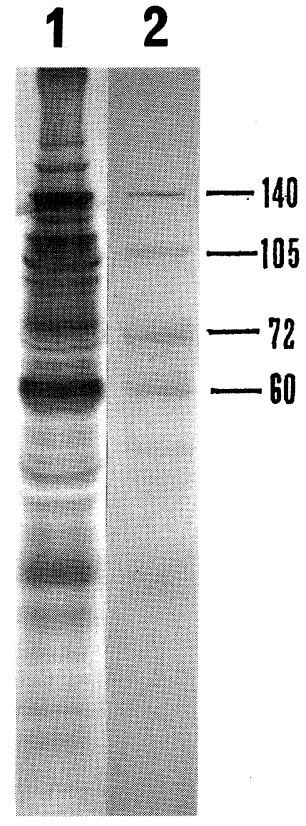

Fig. 13. Characterization of the hemin-binding proteins of pseudonuclei by western blotting analysis using biotin-Con A. The heminbinding proteins isolated from pseudonuclei by hemin-agarose affinity column were resolved by SDS-PAGE and processed for western blotting procedure with biotin-Con A as described under Materials and Methods. Lane 1 desplays total proteins of pseudonuclei, and lane 2 shows the hemin-binding proteins. The prominent Con $\mathrm{A}$ binding proteins were expressed as four bands as indicated by molecular masses. These Con A binding protein bands disappeared when methyl- $\alpha$-D-mannopyranoside was added as a competitor to the solutions for western blotting.

clear reconstitution in the Xenopus interphase egg extract presumably through interactions with several essential proteins. Because porphyrins are strongly hydrophobic, it is reasonable to assume that hemin is involved in hydrophobic interactions with these proteins in the nuclear reconstitution pathway. Based on the configuration and nucleosome structure of sperm chromatins incubated in egg extract containing hemin, we concluded that hemin inhibits nuclear reconstitution at the step of nuclear membrane reconstitution during the swelling process but not at the step of decondensation.

As shown in Fig. 4, the sperm chromatins were likely to become fragile and tender under hemin inhibition. This abnormality became more pronounced as hemin concentration increased. This fragility might result in part from a deficiency in the continuous nuclear envelope formation. The reason for this deficiency has yet to be determined, but the lack of B-type lamin incorporation may be related to the inhibition of continuous nuclear envelope formation. However, it has been shown that chromatin with intact, continuous doublelayered nuclear membrane is formed in egg extract de- pleted of the B-type lamin LIII (23) or in the presence of WGA (7). As reported previously (31), we also observed that the double-layered nuclear membrane deficient of B-type lamin was continuous and showed no sign of fragmentation of nuclear membrane in the nuclei reconstituted in the presence of WGA. Thus the lack of B-type lamin LIII incorporation alone might not result in the fragmentation of membrane observed in this study. On the other hand, Lourim et al. (19) recently found that lamina structure formed in the Xenopus egg extracts is constituted from three B-type lamins (LI, LII, LIII) which are associated with different vesicle populations. In these lamin B species, lamin LIII is a major type. At present the detailed participation in the reconstitution of nuclear envelope in the egg extract remains to be determined. Thus, the process to produce the membrane patch structures might be studied further in the light of the participation of vesicle populations bearing different subtypes of B-type lamin on the reconstitution of nuclear envelope.

As many membrane patches were found on the hemin-blocked chromatins, a part of essential process of the nuclear envelope reconstitution was impaired by hemin. Hemin intercalates into biological membrane and induces membrane disorder $(10,27)$. Thus, the effect of hemin on the nuclear envelope reconstitution process may be generated by the intercalation of hemin into the membrane vesicles of egg extract that were endowed to form nuclear membrane. Alternatively, since the flattened membrane patches and nuclear pores were formed under the inhibition by hemin, the nuclear membrane-associated proteins may be directly inhibited to work on the completion of nuclear envelope reconstitution by hemin. The fact that some of the hemin-binding proteins bound Con A seems to be important to verify the second possibility because several studies have demonstrated lectin binding to nuclear membrane (12, for review). Although we do not know the localization of these Con A-binding proteins in pseudonuclei, the step when the proteins are incorporated into chromatins shown in this study seems to suggest further their role in the reconstitution of nuclear envelope. Thus, future experiments will be especially aimed at correlating each hemin-binding protein with the nuclear envelope reconstitution process.

In conclusion, results from this study provide evidence for the unique inhibitory effect of hemin and isolation of hemin binding proteins which accumulate in the pseudonuclei at the swelling step of nuclear reconstitution in egg extract. Understanding the role of these new types of proteins in nuclear reconstitution will clearly aid in further elucidating the process of nuclear reconstitution and the mechanism of nuclear function. In this line of study, experiments are presently under way to characterize the hemin-binding proteins incor- 
porated to the sperm chromatins.

Acknowledgments. Part of this work was performed under the Frontier Research Project in Telecommunications supported by the Ministry of Posts and Telecommunications and the Support Center for Advanced Telecommunications Technology Research and was supported by a Grant-in-Aid for Science Research from the Ministry of Education, Science and Culture of Japan.

\section{REFFERENCES}

1. Adachi, Y. and LaEmmLI, U.K. 1992. Identification of nuclear pre-replication centers poised for DNA synthesis in Xenopus egg extracts: Immunolocalization study of replication protein A. J. Cell Biol., 119: 1-15.

2. Almaouzini, G. and Wolffe, A.P. 1993. Nuclear assembly, structure, and function: The use of Xenopus in vitro systems. Exp. Cell Res., 205: 1-15.

3. Blow, J.J. and LASKEY, R.A. 1986. Initiation of DNA replication in nuclei and purified DNA by a cell-free extract on Xenopus egg. Cell, 47: 577-587.

4. Chen, J.J. and London, I.M. 1981. Hemin enhances the differentiation of mouse 3T3 cells to adipocytes. Cell, 26: 117122.

5. Dabauvalle, M.-C., Loos K., and Scheer, U. 1990. Identification of a soluble precursor complex essential for nuclear pore assembly in vitro. Chromosoma, 100: 56-66.

6. Dwarki, V.J., Francis, V.N.K., Bhat, G.J., and Padmanaban, G. 1987. Regulation of cytochrome P-450 messenger RNA and apoprotein levels by heme. J. Biol. Chem., 262: 16958-16962.

7. Finlay, D.R. and Forbes, D.J. 1990. Reconstitution of biochemically altered nuclear pores: transport can be eliminated and restored. Cell, 60: 17-29.

8. Gasser, S.M., Ohashi, A., Daum, G., Bohni, P.C., Gibson, J., Reid, G.A., Yonetani, T., and Schatz, G. 1982. Imported mitochondrial proteins cytochrome $\mathrm{b}_{2}$ and cytochrome $\mathrm{c}_{1}$ are processed in two steps. Proc. Natl. Acad. Sci. U.S.A., 79: 267271.

9. Gerace, L. and Burke, B. 1988. Functional organization of the nuclear envelope. Annu. Rev. Cell Biol., 4: 335-374.

10. Ginsburg, H. and Demel, R.A. 1984 . Interactions of hemin, antimalarial drugs and hemin-antimalarial complexes with phospholipid monolayers. Chem. Phys. Lipids, 35: 331-347.

11. Goldberg, M., Jenkins, H., Allen, T., Whitfield, W.G.F., and Hutchison, C.J. 1995. Xenopus lamin B3 has a direct role in the assembly of a replication competent nucleus: evidence from cell-free extracts. J. Cell Biol., 108: 3451-3461.

12. Hart, G.W., Haltiwanger, R.S., Holt, G.D., and Kelly, W.G. 1989. Glycosylation in the nucleus and cytoplasm. Annu. Rev. Biochem., 58: 841-874.

13. Higashiura, M., TaKasuga, Y., Yamashita, J., and Yagura, T. 1993. A protein homologous to human Ku p70-protein is required for reconstitution of Xenopus sperm pronuclei. Chromosome Res., 1: 27-36.

14. Hinshaw, J.E., Carragher, B.O., and Milligan, R.A. 1992. Architecture and design of the nuclear pore complex. Cell, 69: 1133-1141.

15. Hutchison, C.J. and Kill, I.R. 1989. Changes in the nuclear distribution of DNA polymerase $\alpha$ and PCNA/cyclin during the progress of the cell cycle, in a cell-free extract of Xenopus eggs. J. Cell Sci., 93: 605-613.

16. Jeong, S.W., Lauderdale, J.D., and Stein, A. 1991. Chromatin assembly on plasmid DNA in vitro. Apparent spreading of nucleosome alignment from one region of pBR327 by his- tone H5. J. Mol. Biol., 222: 1131-1147.

17. Kubota, Y., Mimura, S., Nishimoto, S., Takisawa, H., and NoJima, H. 1995. Identification of the yeast MCM3-related protein as a component of Xenopus DNA replication licensing factor. Cell, 81: 601-609.

18. LOHKA, M.J. and MALLER, J.L. 1985. Induction of nuclear envelope break down, chromosome condensation, and spindle formation in cell-free extracts. J. Cell Biol., 101: 518-523.

19. Lourim, D., KempF, A., and Krohne, G. 1996. Characterization and quantitation of three B-type lamins in Xenopus oocytes and eggs: Increase of lamin L1 protein synthesis during meiotic maturation. J. Cell Sci., 109: 1775-1785.

20. Marks, P.A., Rafkind, R.A., Gambari, R., Epner, E., Chen, S.-X., and BANKS, J. 1982. Commitment to terminal differentiation and the cell cycle. Curr. Top. Cell. Regul., 21: 189-203.

21. Mazoy, R., Vzquez, F., and Lemos, M.L. 1996. Isolation of heme-binding proteins from Vibrio anguillarum using affinity chromatography. FEMS Microbiol. Lett., 141: 19-23.

22. MeIer, E., Miller, B.R., and Forbes, D.J. 1995. Nuclear pore complex assembly studied with a biochemical assay for annulate lamellae formation. J. Cell Biol., 129: 1459-1472.

23. Newport, J.W., Wilson, K.L., and Dunphy, W.G. 1990. A lamin-independent pathway for nuclear envelope assembly. $J$. Cell Biol., 111: 2247-2259.

24. ОсноА, S. 1983. Regulation of protein synthesis initiation in eukaryotes. Arch. Biochem. Biophys., 223: 325-349.

25. Panté, N. and Aebi, U. 1993. The nuclear pore complex. $J$. Cell Biol., 122: 977-984.

26. Philipott, A. and Leno, G.H. 1992. Nucleoplasmin remodels sperm chromatin in Xenopus egg extracts. Cell, 69: 59-767.

27. Schmitt, T.H., Frezzatti, W.A., JR., and Schreier, S. 1993. Hemin-induced lipid membrane disorder and increased permeability: A molecular model for the mechanism of cell lysis. Arch. Biochem. Biophys., 307: 96-103.

28. Sheehan, M.A., Mills, A.D., Sleeman, A.M., Laskey, R.A., and Blow, J.J. 1988. Steps in the assembly of replication-competent nuclei in a cell-free system from Xenopus eggs. J. Cell Biol., 106: 1-12.

29. Solar, I. and Shaklai, N. 1989. Association of hemin with protein 4.1 as compared to spectrin and actin. Biochem. Biophys. Acta, 983: 199-204.

30. Takasuga, Y., Andoh, T., Yamashita, J., and Yagura, T. 1995. ICRF-193, an inhibitor of topoisomerase II, demonstrates that DNA replication in sperm nuclei reconstituted in Xenopus egg extracts does not require chromatin decondensation. Exp. Cell Res., 217: 378-384.

31. Takasuga, Y., Morita, M., Yamashita, J., Andoh, T., and YAGURA, T. 1995. Aphidicolin-sensitive DNA polymerase is incorporated into the chromatin during nuclear envelope assembly in Xenopus egg extract. Exp. Cell Res., 219: 283-291.

32. TAKasuga, Y. and Yagura, T. 1993. Assembly of envelope structure with vesicles associated with $\mathrm{Ku}$-homologous protein in Xenopus egg extract in the absence of chromatin. Cell Struct. Funct., 18: 205-210.

33. Yagura, T., Kozu, T., and Seno, T. 1987. Immunochemical detection of a primase activity related subunit of DNA polymerase $\alpha$ from human and mouse cells using the monoclonal antibody. Biochemistry, 26: 7749-7754.

34. Vierstra, R.D. and Sullivan, M.L. 1988. Hemin inhibits ubiquitin-dependent proteolysis in both a higher plant and yeast. Biochemistry, 27: 3290-3295.

(Received for publication, April 23, 1998

and in revised form, August 5, 1998) 